(1)

CrossMark

\title{
To compare the incomparable: COVID-19 pneumonia and high-altitude disease
}

\author{
To the Editor:
}

The coronavirus disease 2019 (COVID-19) pandemic is overwhelming healthcare systems worldwide. There is no evidence from randomised clinical trials that any potential therapy improves outcome in COVID-19 pneumonia, and therapeutic strategies have been based on a progressively increasing knowledge of the clinical presentation of the disease. Some clinicians have found the clinical features of COVID-19 pneumonia to be similar to high-altitude pulmonary oedema (HAPE) [1], and such theory has been amplified via social media. We question this relationship.

COVID-19 pneumonia shows diffuse alveolar damage and airway inflammation, reflecting a true virus-related pathology [2], as shown by the first report of pathological findings from autopsies in COVID-19 decedents published at the beginning of April 2020 [1]. Since the end of February 2020, as clinicians from northern Italy and other European countries, we have faced the management of several hundreds of critically ill patients, similarly to other colleagues [3, 4]. Most of the patients with COVID-19 pneumonia who require hospitalisation and intensive care meet the acute respiratory distress syndrome (ARDS) criteria specified in the Berlin definition, but with an unusual clinical manifestation. Hypoxaemia is the main characteristic of all patients with COVID-19 pneumonia and ARDS, but some patients run an unusual course of the disease and show specific abnormalities on chest imaging. These patients show a dissociation between relatively well-preserved lung mechanics and the severity of hypoxaemia. In contrast to classic ARDS, lung volume and dead space in COVID-19 patients are often normal, consolidations absent and pulmonary and chest wall elastance preserved, but a severe capillary leak dominates the initial phase. Whether this severe leak is secondary to virus-triggered inflammation, endothelial cell injury, or direct virus-induced cell destruction, is the subject of ongoing trials. GATTINONI et al. [5] have hypothesised two different phenotypes of COVID-19 pneumonia: a phenotype L, characterised by low elastance, low recruitability, low response to positive end-expiratory pressure (PEEP) and low ventilation/perfusion matching; and a phenotype $\mathrm{H}$ (similar to classic ARDS) with high elastance, higher shunt, high recruitability and higher PEEP response. Despite the assumption that patients may progress from phenotype $\mathrm{L}$ to phenotype $\mathrm{H}$ over time, and that this evolution could be disease- and/or therapy-driven, further evidence is needed to prove this hypothesis.

The assumption that the clinical features of COVID-19 pneumonia are similar to HAPE [1] may rely on the initial clinical presentation of COVID-19 patients, showing profound hypoxaemia with no respiratory distress, similar to patients with acute high-altitude disease that have a chemoreceptor dysfunction. HAPE is a non-cardiogenic pulmonary oedema caused by exaggerated hypoxic pulmonary vasoconstriction and abnormally high pulmonary artery pressure and capillary pressure [6]. These high pressures lead to a non-inflammatory and haemorrhagic alveolar capillary leak in the earlier stages of the disease [7]. Over time, haemorrhagic alveolar capillary leak may also evoke a secondary inflammatory response in HAPE patients [7]. The pathogenesis of the two diseases (HAPE and COVID-19 pneumonia) is clearly different, despite similarities in clinical features, chest imaging and bronchoalveolar lavage findings in later stages, as has recently been emphasised by LuKs et al. [8]. Due to the different pathogenesis, adoption of prevention and treatment strategies from HAPE for the treatment of patients with COVID-19 pneumonia could lead to potential pitfalls. Patients with COVID-19 pneumonia often require intensive care, whereas HAPE is a life-threatening condition that almost never needs intensive care, as it can be reversed by oxygen administration, exposure to a relatively hyperbaric environment or transfer to lower altitude [9]. The use

@ERSpublications

COVID-19 pneumonia is a viral infection; high-altitude pulmonary oedema is a non-cardiogenic oedema. Some clinicians have found the clinical features similar. It is important to clarify such misconceptions to prevent erroneous treatment strategies https://bit.ly/2KOBi3F

Cite this article as: Strapazzon G, Hilty MP, Bouzat P, et al. To compare the incomparable: COVID-19 pneumonia and high-altitude disease. Eur Respir J 2020; 55: 2001362 [https://doi.org/10.1183/ 13993003.01362-2020]. 
of supplemental oxygen in COVID-19 pneumonia does not counteract the underlying pathogenic mechanisms in the way it does in HAPE [9], but only increases oxygen availability. Systemic pulmonary vasodilators such as nifedipine and phosphodiesterase-5 inhibitors could critically worsen ventilation/ perfusion mismatch or cause hypotension in COVID-19 patients, where there is no hypoxic pulmonary vasoconstriction like in HAPE [6] but rather a dysregulation of lung perfusion and impaired hypoxic vasoconstriction. Despite its established role in prevention and treatment of HAPE [9], corticosteroid use in COVID-19 pneumonia is still under debate in the scientific community [10] and currently not advised by the World Health Organization.

We consider it important to clarify such misconceptions, in order to prevent the spreading of erroneous treatment strategies when caring for COVID-19 patients. COVID-19 disease has been reported to affect high-altitude areas [11] and, interestingly, the spread of the disease in certain areas of northern Italy has started from villages in mountainous areas that are popular with tourists. This does not implicate any coincidence between the diseases. Clinicians should focus on the development of therapeutic strategies based on the pathogenesis of the disease, and should remember that COVID-19 pneumonia is a viral infection primarily leading to diffuse alveolar damage and airway inflammation.

Giacomo Strapazzon ${ }^{1,2}$, Matthias P. Hilty $\oplus^{3}$, Pierre Bouzat $^{4}$, Lorenza Pratali $^{5}$, Hermann Brugger ${ }^{1,2}$ and Simon Rauch (10)

${ }^{1}$ Institute of Mountain Emergency Medicine, Eurac Research, Bolzano, Italy. ${ }^{2}$ Dept of Anaesthesiology and Critical Care Medicine, Medical University of Innsbruck, Innsbruck, Austria. ${ }^{3}$ Intensive Care Unit, University Hospital of Zurich, Zurich, Switzerland. ${ }^{4}$ Dept Anaesthesia and Critical Care, University Hospital of Grenoble, Grenoble, France. ${ }^{5}$ Institute of Clinical Physiology, National Council of Research - CNR, Pisa, Italy. ${ }^{6}$ Dept of Anesthesia and Intensive Care Medicine, "F. Tappeiner" Hospital, Merano, Italy.

Correspondence: Giacomo Strapazzon, Institute of Mountain Emergency Medicine, Eurac Research, Via Ipazia 2, 39100 Bolzano, Italy. E-mail: giacomo.strapazzon@eurac.edu

Received: 23 April 2020 | Accepted after revision: 27 April 2020

Conflict of interest: G. Strapazzon has nothing to disclose. M.P. Hilty has nothing to disclose. P. Bouzat has nothing to disclose. L. Pratali has nothing to disclose. H. Brugger reports personal fees from Eurac Research, outside the submitted work. S. Rauch has nothing to disclose.

\section{References}

1 Solaimanzadeh I. Acetazolamide, nifedipine and phosphodiesterase inhibitors: rationale for their utilization as adjunctive countermeasures in the treatment of coronavirus disease 2019 (COVID-19). Cureus 2020; 12: e7343.

2 Barton LM, Duval EJ, Stroberg E, et al. COVID-19 autopsies, Oklahoma, USA. Am J Clin Pathol 2020; 153: 725-733.

3 Gattinoni L, Coppola S, Cressoni M, et al. COVID-19 does not lead to a "typical" acute respiratory distress syndrome. Am J Respir Crit Care Med 2020; 201: 1299-1300.

4 Grasselli G, Zangrillo A, Zanella A, et al. Baseline characteristics and outcomes of 1591 patients infected with SARS-CoV-2 admitted to ICUs of the Lombardy region, Italy. JAMA 2020; 323: 1574-1581.

5 Gattinoni L, Chiumello D, Caironi P, et al. COVID-19 pneumonia: different respiratory treatments for different phenotypes? Intensive Care Med 2020; in press [https://doi.org/10.1007/s00134-020-06033-2].

6 Maggiorini M, Mélot C, Pierre S, et al. High-altitude pulmonary edema is initially caused by an increase in capillary pressure. Circulation 2001; 103: 2078-2083.

7 Swenson ER, Maggiorini M, Mongovin S, et al. Pathogenesis of high-altitude pulmonary edema: inflammation is not an etiologic factor. JAMA 2002; 287: 2228-2235.

8 Luks A, Freer L, Grissom C, et al. COVID-19 lung injury is not high altitude pulmonary edema. High Alt Med Biol 2020; in press [https://doi.org/10.1089/ham.2020.0055]

9 Bärtsch P, Swenson ER. Clinical practice: acute high-altitude illnesses. N Engl J Med 2013; 368: 2294-2302.

10 Russell CD, Millar JE, Baillie JK. Clinical evidence does not support corticosteroid treatment for 2019-nCoV lung injury. Lancet 2020; 395: 473-475.

11 Zeng J, Peng S, Lei Y, et al. Clinical and imaging features of COVID-19 patients: analysis of data from high-altitude areas. J Infect 2020; 80: e34-e36. 